\title{
The role of self-efficacy in cardiovascular disease self-management: a review of effective programs
}

This article was published in the following Dove Press journal:

Patient Intelligence

4 October 2010

Number of times this article has been viewed

\author{
Hannah Katch \\ Holly Mead \\ The George Washington University, \\ Department of Health Policy, \\ Washington DC, USA
}

Correspondence: Hannah Katch The George Washington University, Department of Health Policy, Washington DC, USA

Tel + I 2029943367

Email hannah.katch@gwumc.edu
Research objective: To conduct a comprehensive, systematic review of disease self-management programs for patients with cardiovascular disease (CVD), looking specifically at those with self-efficacy as a key component to the effectiveness of such programs on CVD management and outcomes.

Study design: We conducted a review of effective strategies promoting patient involvement and engagement in the self-management of CVD. To narrow the scope of the review, we defined strategies that were empirically tested and showed a measurable and positive impact on outcomes that reflect improved self-management (eg, medication adherence or patient's perceived management skills) and/or improved clinical outcomes (eg, lower blood pressure or reduced hospitalization).

Results: We identified five disease management programs focusing on self-efficacy that had been rigorously evaluated by multiple studies in varying patient populations. Each of these programs were shown to be effective in increasing patients' engagement and involvement in the management of their disease, by demonstrating improvement in self-efficacy while developing patients' self-management skills. They also were shown to improve clinical outcomes for patients, such as lower blood pressure and reduced hospitalizations.

Conclusion: The studies that we reviewed demonstrate the importance of including selfefficacy as a key component in CVD self-management programs. These programs should be multidisciplinary in approach, should be tailored to the needs of the patients, and should have a theoretical foundation of behavior change. More research is needed to investigate the causal link between self-efficacy, self-management and clinical outcomes.

Keywords: cardiovascular disease (CVD), self-management, self-efficacy

\section{Introduction}

\section{The problem of cardiovascular disease}

Cardiovascular disease (CVD) is the leading cause of death for adults in the United States, and is responsible for more than $40 \%$ of annual deaths. ${ }^{1}$ Approximately 80 million, or one in three individuals, have one or more types of CVD. ${ }^{2}$ In 2005 , the overall death rate from CVD was 278.9 per $100,000 .^{2}$ The problem of CVD in the United States is more substantial than in other developed countries. For example, the World Health Organization reports that the United States has among the highest rate of disabilityadjusted life years (DALYS) due to CVD, 8 DALYS per 1000 people $^{3}$ in comparison to other developed countries.

There are considerable differences in the death rates for males and females in the United States, and between different racial and ethnic groups. Black men and women have higher death rates than white men and women, and men have higher overall rates 
of mortality from the disease regardless of race. Since 1950 , the CVD death rate has declined 66\%, ${ }^{1}$ and between 1995 and 2005, death rates from CVD declined $26.4 \%{ }^{2}$

CVD mortality is associated with modifiable risk factors such as diabetes, high cholesterol, high blood pressure, smoking, obesity, dietary factors and physical inactivity. ${ }^{2}$ Socioeconomic status and other nonmodifiable risk factors are also associated with CVD mortality. ${ }^{1}$ Heart disease also results in substantial health care expenditures. ${ }^{4}$ Three of the most expensive principal procedures in the United States are cardiac-related ${ }^{1}$ and coronary heart disease alone cost an estimated $\$ 151.6$ billion in direct and indirect costs in the United States in $2007 .{ }^{4}$ In addition to mortality, poor management of CVD can lead to long-term disability due to complications from acute myocardial infarctions (AMI), strokes, congestive heart failure, and end-stage renal disease. ${ }^{5}$

\section{The role of self-management in CVD control}

A key factor contributing to the decline in CVD mortality over the past 60 years is a better control of risk factors that contribute to CVD. ${ }^{1}$ The determinants of health status in patients with CVD are still being identified, particularly the impact of self-efficacy on clinical outcomes. However, the literature has shown that patients with better control of their care are often more educated about their disease and more engaged in their own management of these risk factors. ${ }^{6}$

According to the literature, self-management refers to an individual's ability to manage symptoms, treatment protocols, physical and psychosocial consequences, and lifestyle changes inherent to living with a chronic condition. ${ }^{7}$ Selfmanagement is unavoidable: individuals make choices about their health behaviors everyday. Effective self-management is an important factor in making good decisions about health behaviors and therefore determining health outcomes. ${ }^{9}$

Research has shown that effective self-management can also improve the clinical outcomes of patients with chronic illness. ${ }^{9}$ According to a study by Corbin and Strauss, ${ }^{10}$ effective self-management has three components. First, patients must manage the medical aspects of their condition, such as following a medication regimen or adhering to a special diet. Second, the patient must maintain, change or create new behaviors based on the challenges or restrictions of their condition, such as changing their exercise or sleeping patterns to adjust for shortness of breath or increased fatigue. And third, the patient must manage the emotional impact of his or her condition, including adjusting future plans and the emotions that may arise due to these changes, such as frustration or anger. ${ }^{10}$
Kate Lorig's extensive work $^{9,11-13}$ in effective selfmanagement shows that these three components rely on patients' abilities to identify problems in their current routines and to develop solutions. In addition to problemsolving, effective self-management involves decision making, resource utilization, forming of a patient/health care provider partnership, and taking action. ${ }^{9}$ Lorig's work has also shown the importance of self-management programs in improving CVD health outcomes. ${ }^{12}$

CVD is usually managed in part with prescription medications. ${ }^{14}$ Optimal health outcomes therefore require a high level of self-management in order to adhere to medication regimens. Poor medication management can lead to increased morbidity and mortality, as well as increased consumption of health care resources. ${ }^{14}$ However, adherence to medication is quite low among CVD patients. One study found that only $50 \%$ to $60 \%$ of patients adhered to prescribed medications over a one-year period. ${ }^{15}$ Medication nonadherence in chronic diseases accounts for $10 \%$ of hospital readmissions in the United States. ${ }^{16}$ While socioeconomic status, cognitive function, and lifestyle are all factors associated with medication self-management, ${ }^{16}$ the literature also suggests that non-adherence to medication regimens is at least in part a consequence of active decision-making on the part of patients. ${ }^{14}$ Patients bring their own perspectives to the use of medication and make decisions based on their beliefs and experiences regarding the perceived efficacy of the medication. ${ }^{14}$ Improving self-efficacy can be a key component in improving patient adherence to medication regimens. An intervention that works to improve patient perception of his or her own ability to adhere to medication regimens can improve clinical outcomes and decrease costs by increasing adherence to medication regimens. ${ }^{14}$

Another important aspect of CVD self-management is lifestyle adjustment. Patients with CVD are often advised by their physicians to increase exercise or participate in cardiac rehabilitation (CR) programs, to change their diet to promote heart health, to lose weight, and/or to quit smoking. ${ }^{17}$ These lifestyle changes, especially in combination with physical and psychological symptoms experienced by many CVD patients, can be overwhelming for patients. ${ }^{17}$ Self-efficacy is an especially important factor in making lifestyle changes, since these changes depend entirely on the daily decisions of the individuals.

\section{Patient engagement and self-efficacy}

Traditional patient education programs have focused on patients changing their behavior based on current 
recommendations for their health status. However, studies have shown that if patients do not believe in their own ability to improve their health status through behavior change, the association between healthful behaviors and health status change is weak. ${ }^{9}$ Further studies have shown that an important mechanism in improving health status for participants in self-management programs is patient self-efficacy, ${ }^{7,9-11,18-20}$ or a patient's engagement and belief in his or her ability to carry out or change behavior necessary to the desired goal. By engaging patients in problem-solving and tailoring disease management skills to their particular challenges, self-management programs can improve patient self-efficacy. Lorig's work shows that engaging patients in their disease management and increasing self-efficacy are critical in linking disease management to improved clinical outcomes. ${ }^{9}$

Studies have also demonstrated that patient engagement and self-efficacy are important factors of disease management in patients with CVD. The literature demonstrates that patients' own perceptions of their ability to self-manage their CVD improve health behaviors and clinical outcomes. ${ }^{6,7,18,20}$

\section{Barriers to effective self-management}

Many patients with CVD experience considerable barriers to self-management. ${ }^{6,17}$ Expensive and time-consuming treatments, long recoveries, rehabilitation, and medication management are significant burdens even for patients with substantial resources, and can be seemingly insurmountable barriers for patients with co-morbidities, few resources and/or insufficient information about their condition. ${ }^{5}$ Physical limitations, difficulty coping with treatment, and emotional distress can also be serious barriers to care for patients with CVD. ${ }^{5}$ Access to care and uninsurance or underinsurance are also established barriers that often disproportionately affect minority patients. ${ }^{17}$

An important factor of disease self-management programs lies in helping patients to overcome the barriers they face in managing their disease. While this is often a multifaceted task, improving patient engagement and self-efficacy is a crucial step in helping patients to take charge of their disease management.

CVD can have emotional and psychosocial effects, as well as physical ones. The literature suggests that many patients with CVD struggle with depression, anxiety, stress and negative social conditions that can lead to increased rates of morbidity and mortality. ${ }^{21}$ These effects can also impact patients' beliefs about their illness and its treatment, which can in turn drive patients' behavior. ${ }^{8,21}$ Interventions that seek to improve patient self-efficacy can be crucial to improving the cognitive state of patients with CVD, and therefore improve their clinical outcomes in addition to their quality of life. ${ }^{21}$

Low patient comprehension and health illiteracy are two additional obstacles that impede patients' ability to effectively manage their CVD. One study of low-income, older adults found that $25 \%$ of participants reported difficulty understanding written information from clinicians. ${ }^{22}$ According to another recent study, patients with inadequate literacy skills are 10 to 18 times more likely to be unable to identify all their medications, compared with those with adequate literacy skills. ${ }^{16}$ Patients with low health literacy have $29 \%$ to $52 \%$ higher hospitalization rates, even after adjustment for baseline socioeconomic status, health status and health behaviors. ${ }^{23}$ Prior research has shown that inadequate health literacy is associated with less knowledge of chronic disease and poor self-management skills for patients with chronic illnesses. $^{23}$

Vulnerable populations such as communities of color, low-income populations and women are also more likely to have many of the risk factors for CVD as well as to experience adverse CVD outcomes. ${ }^{24}$ Physician encouragement for self-management is particularly important for these patient groups. Despite the disparities in health outcomes, there is evidence that some physicians are less likely to provide information about healthy behaviors to non-white patients than to whites, ${ }^{25,26}$ and are less patient-centered with nonwhite patients. ${ }^{27}$ One possible explanation for this difference is the observed vulnerability of minority patients to lower confidence to self-manage, ${ }^{27}$ which would exacerbate existing racial and ethnic disparities in CVD outcomes.

The evidence clearly suggests the importance of selfefficacy as a component of self-management and that barriers impede patients from effectively managing CVD. Therefore, the purpose of this review is to identify those programs that explicitly focus on self-efficacy and have been shown to have statistically significant improvement in CVD outcomes. Through our systematic review, we developed criteria to identify the programs most relevant to our objective and examined the scientific evidence collected in our review. Our focus on the role of self-efficacy within self-management programs for patients with CVD fills an important gap in the existing literature. No other review to date has shown different self-management approaches that quantitatively correlate self-efficacy with improved health outcomes.

\section{Methods}

Through a systematic review of current literature on management of CVD, we aim to clearly identify the 
importance of patient self-efficacy in the everyday management of chronic illness and the effectiveness of selfmanagement programs that integrate self-efficacy support. Through our comprehensive search, we developed criteria to examine the programs most relevant to our objective and examined the scientific evidence collected in our systematic review. Based on the premise that self-efficacy is a critical component in self-management programs, we looked specifically at strategies promoting patient involvement and engagement, or those strategies that focused explicitly on self-efficacy and patient activation among CVD patients. We defined self-efficacy as a patient's engagement and belief in his or her ability to carry out or change behavior necessary to the desired goal. Given the extent of CVD in the United States, ${ }^{3}$ we also limited our review to those programs and studies conducted in the United States since 1995. The current time frame allowed for a review of programs that are currently in use and therefore more relevant to the field of CVD management in the United States.

\section{Data sources and searches}

To identify articles that met our criteria, we conducted comprehensive searches on PubMed and Scopus in April, 2010 using predetermined keywords (see Figure 1). We also examined references cited by recently published, relevant articles, to identify studies that may not have come up in our keyword search. Finally, for more established programs, such as the Chronic Disease Self-Management Program (CDSMP), we searched program websites for additional evaluations testing the intervention.

\section{Article selection}

After reviewing almost 200 studies and interventions (see Figure 1), we narrowed our scope to five peer-reviewed programs that have been rigorously evaluated in the United States since 1995. The programs we include in this review focus on strategies and interventions to promote patient selfefficacy in CVD management. We only include programs that measure and empirically evaluate patients' self-efficacy, and that show a measurable and positive impact on outcomes that reflect improved self-management (eg, medication adherence or patient's perceived management skills) and/or improved clinical outcomes (eg, lower blood pressure or reduced hospitalization). We paid close attention to identifying articles with interventions that included patients from vulnerable populations including low literacy populations and racial and ethnic minorities.

\section{Review process}

We managed the review process by inputting summaries of relevant articles into an Excel spreadsheet (Microsoft, Redmond, WA) that identified the database source, the relevant search terms, the programs tested and the outcomes measured. We describe each of the programs ultimately identified in our review, emphasizing the role of self-efficacy in each one, and we note the improvement that the programs had on specific outcomes. We also identify common components in each of the programs that improved their success rates and make recommendations for future self-management program development and further research.

\section{Results}

We identified a total of five self-management programs that met our criteria and focused on activating CVD patients and improving self-efficacy in an effort to better engage patients in their care and to improve overall outcomes. The interventions tested in the following studies demonstrate improvements in patient activation and self-efficacy, as well as improved clinical outcomes, including lower blood pressure and reduced hospital admissions.

The first two programs that we identify in this review focus on Lorig's foundational work in the development of disease self-management programs. These studies emphasize her findings that self-efficacy is a critical component of managing a chronic disease. The first program is the CDSMP, one of the first programs designed to assist with chronic disease self-management. The second program, Tomando Control de su Salud (Take Control of your Health) was developed as a sister program to the CDSMP, but with a specific emphasis on the unique challenges faced by non-English-speaking patients. Specific, tailored components were added to the Tomando program to address these needs. The third program we identified is the "Women Take PRIDE" program, which focuses on the self-management issues that older women have in controlling their disease. Our fourth program looks at the effect of CR on exercise, recognizing the effect of exercise of self-efficacy. The final program we discuss is a disease management program tailored to the needs of low literacy populations.

\section{Program I}

The first program we identified was the CDSMP, a widelystudied program developed by Lorig and colleagues that uses self-efficacy theory to improve disease management. ${ }^{11-13,28-33}$ This program was developed to help patients with chronic diseases develop self-management skills. The intervention 


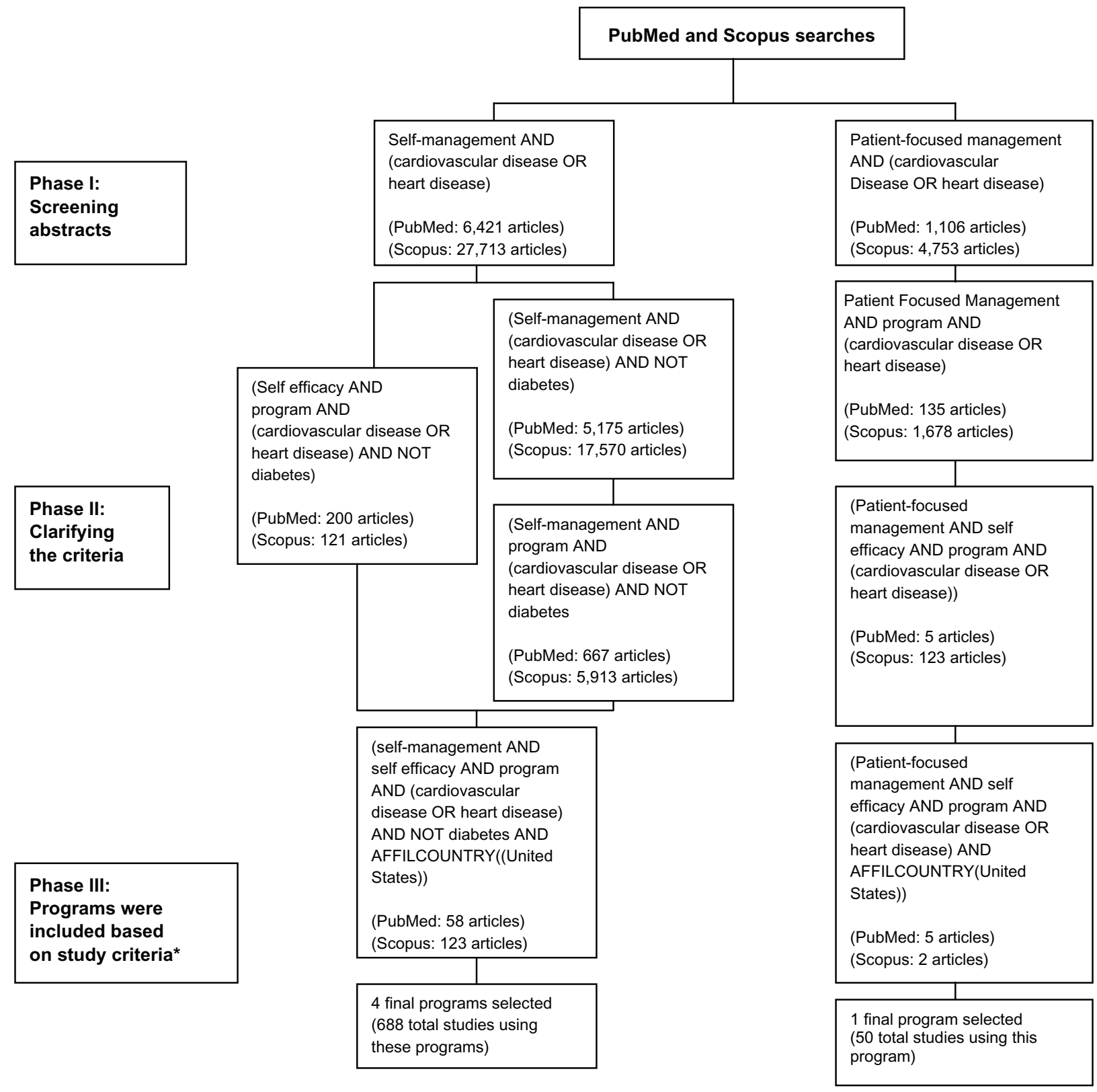

Figure I Article review flowchart on self-efficacy in CVD self-management programs. Note: *as discussed in data management section.

consists of a seven-week group program with weekly 2.5 hour sessions on issues such as adopting exercise programs; using cognitive symptom management techniques; changing dietary habits; adhering to medications; using available community resources; managing fear, anger and depression; and trainings on effective communication with health professionals. ${ }^{11}$

The program developers evaluated the CDSMP initially with a six-month randomized, controlled trial and again with a follow-up, longitudinal study of the randomized trial. The initial study compared six-month outcomes of the treatment group $(n=644)$ and the control group $(n=476)$ on three categories of outcome variables: health behaviors, health status and health service utilization. Specific measures used for health status included self-rated health, disability, social/role activities, limitations, pain/physical discomfort, psychological well-being, energy/fatigue, health distress, shortness of breath. Health behavior measures reported in the study were stretching/strengthening exercise, aerobic exercise, cognitive symptom management and communication with physician. Health services utilization outcomes included outpatient and emergency room (ER) visits, number of hospital stays (past six-months) and nights in the hospital 
(past six-months). All measures used were either validated measures from prior studies or were developed and tested for reliability and validity for the evaluation. ${ }^{34}$ The study authors used analysis of covariance on endpoint scores to compare six-month outcomes between the treatment and control groups, controlling for both baseline values of the study variable and demographic variables.

After six months, the treatment group demonstrated significant improvement in all four health behaviors and five of the health status outcomes compared to the control group $(P<0.05)$. Specifically, participants in the treatment groups had significantly higher six-month increases in minutes of stretching/strengthening exercise (13 more minutes/week for the treatment group versus five more minutes/week for the control group) and aerobic exercise (16 additional minutes/ week versus two fewer minutes/week, respectively). They also had significantly higher changes in cognitive symptom management scores $(0.38$ change for the treatment group on a $0-5$ point scale versus a 0.07 increase for the control group) and communication with physician scores ( 0.26 change versus 0.11 change, respectively, on a $0-5$ point scale) than the control group. ${ }^{11}$

The treatment group also showed significant improvement in several health status outcome measures compared to the control group $(P<0.05)$. Participants in the treatment group had significantly higher self-rated health status than members of the control group (an improvement of 0.09 as compared to a decline of 0.02 , respectively, on a $1-5$ point scale) and the treatment group had a mean decrease in disability as compared to those in the control group $(-0.02$ as compared to 0.03 , respectively, on a $0-3$ point scale). Participants in the treatment group also reported an improvement in ability to fulfill social/role activities (a 0.07 improvement as compared to a 0.08 decline in the control group, on a 0-4 point scale), and an improvement in psychological well being ( 0.09 in the treatment group compared to 0.04 in the control group, on a $0-5$ point scale). The treatment group also reported improved energy as compared with the control group ( 0.14 improvement in the treatment group versus 0.02 in the control group, on a $0-5$ point scale) and a reduction in health distress ( 0.24 versus 0.07 reduction respectively, on a $0-4$ point scale). ${ }^{11}$

Treatment group participants also reported fewer hospital stays $(0.07$ fewer in the treatment group versus 0.05 in the control group in the past six-months), and fewer nights in the hospital $(0.28$ fewer in the treatment group versus 0.56 more in the control group in the past six-months). ${ }^{11}$

The CDSMP researchers also conducted a follow-up, longitudinal study of the randomized trial to examine the effectiveness of the program at one and two years on three categories of outcomes: health status, health services utilization and perceived self-efficacy. ${ }^{12}$ The health status measures reported on in the follow-up study were self-rated health, disability, social/role activities limitations, energy/fatigue and health distress. The health services utilization measures included physician/ER visits, number of hospitalizations and days in the hospital. The self-efficacy measure used in this study combined two scales that had been developed and validated for the CDSMP and measured perceived adaptability to manage different aspects of chronic disease, such as pain and fatigue. The self-efficacy measure had a chronbach's $\alpha$ coefficient of $0.89 .^{12}$

The researchers used matched-pair $t$-tests to test for changes in health status, health services utilization and self-efficacy. All participants in the study participated in the CDSMP program and outcomes were measured at baseline and one or two years. The study found that participants displayed significant reduction in health distress $(-0.199$ change at year 1 on a $0-5$ point scale and -0.290 change at year 2; $P<0.0001$ ), demonstrated an increase in perceived self-efficacy ( 0.310 change on a $0-10$ point scale at year one and 0.270 change at year two; $P<0.0001$ ), and made fewer visits to physicians and the emergency department ( 0.689 fewer visits at year one and -0.564 fewer visits at year two; $P<0.05$ ) at each follow-up period as compared to their baseline status. ${ }^{28}$ While the participants had an average 2.2 chronic conditions and higher disability after two years compared to baseline, they did not show deterioration in any other health status variables over the two years of the study $(P<0.05)$, contrary to expectations. ${ }^{12}$

Noting the increase in self-efficacy and decrease in health services utilization, the study authors also conducted a series of multiple regressions to determine the effects of self-efficacy on outpatient use. The researchers found that reduced utilization at year one was significantly associated with higher levels of self-efficacy at year one $(P<0.0001)$ and that larger improvements in self-efficacy at six-months is significantly associated with even lower utilization at year one $(P=0.0203) .{ }^{12}$

Finally, the researchers note that in addition to the program's impact on clinical outcomes, these results have significant health care cost implications as well. During each year, participants made approximately 2.5 fewer visits to emergency departments and physicians than in the previous year, despite their increase in disability over the course of the study. The researchers calculate that at minimum, the savings in reduced outpatient visits were $\$ 100$ per participant. Participants in the study also had an average 0.49 fewer days of 
hospitalization than control group subjects. The researchers calculate approximately $\$ 490$ less utilization per participant at baseline. ${ }^{12}$

Since the development of this program over 10 years ago, it has been extensively used and tested in patients with a number of different chronic diseases across the world. Over 100 studies testing the CDSMP have been conducted, including more than 10 with cardiac patients specifically. The overwhelming results of these studies have shown positive change in patients' behaviors and health status.

\section{Program 2}

Another program developed by Lorig and colleagues focused on disease management for Spanish-speaking patients. Tomando Control de su Salud ${ }^{35}$ (Taking Control of Your Health) seeks to improve patient self-efficacy through a community-based program for Spanish-speakers with chronic diseases including heart disease. The intervention is a 14-hour community-based program presented in 2.5-hour sessions over six weeks. While theoretically based on the CDSMP, Tomando Control de su Salud was developed to be culturally and linguistically appropriate for Spanish-speaking patients, taking into consideration unique challenges faced by this patient population. The program is not just a translation from the English CDSMP program, but includes specific concepts, components and processes developed with extensive input from Spanish-speaking patients with heart disease or other chronic conditions and health professionals. Using the information gained from these patients through focus group discussions, the researchers made cultural adaptations of concepts and content from the CDSMP. ${ }^{35}$ The goal of the program is to improve patients' self-management skills, health status and health care utilization.

Two trained peer leaders guided the sessions in community-based settings such as churches and neighborhood centers. The classes include material on healthy eating, selection of food products, portion size and meal planning. Activities also include weekly action plans to enhance skills mastery, discussion of the meaning and causes of common symptoms to assist with the reinterpretation of these symptoms. Additional sections include relaxation techniques and a section on family communications. Exercise was also performed in class sessions using culturally appropriate audiotapes. The participants also received a book containing the program content, as well as detailed disease-specific information and an exercise tape. ${ }^{35}$

The initial evaluation of the program examined four outcome measures. These measures were based on the priorities identified by the participant focus groups and the theoretical foundation of the study. The measures included health behaviors, health status, health care utilization and self-efficacy. Health behavior outcome measures included number of minutes spent in exercise per week, communication with a physician, mental stress management, and current tobacco use. The health status outcome measures included self-reported health, health distress, fatigue, pain or physical discomfort, and ability to perform one's role. Health care utilization outcome measures examined the number of times in the past four months that participants had visited a physician, visited the emergency department, and visited the hospital. The four-item self-efficacy scale asked participants their confidence in how well they can control the fatigue, pain, emotional distress, and other symptoms caused by their disease in order to perform daily activities. ${ }^{35}$

The study included a control group of 224 individuals and a treatment group of 327 for a four-month randomized study. For this portion of the study, a questionnaire was completed by 265 treatment participants (81\%) and 178 of the control group subjects (79\%) and the study authors used analysis of covariance to compare the intervention and control groups for each outcome variable, controlling for age, gender, education, acculturation, and number of chronic conditions. The study authors also completed a one-year longitudinal study using a paired $t$-test to compare outcomes of participants in the intervention group with baseline. ${ }^{35}$

Four months after program completion, participants' selfefficacy, health behaviors and health status had improved, as compared to baseline. On a 10-point scale, self-efficacy had increased in the treatment group by 1.157 from baseline, as compared to a change of 0.719 for the control group $(P=0.006)$. Participants in the treatment group also showed clear improvement in health status measures from baseline and in comparison with the control group. The self-reported health status of participants in the treatment group, measured on a scale of $0-23$, improved by 0.392 from baseline, as compared to control group, whose self-reported health status improved by 0.028 from baseline $(P<0.0001)$. The treatment group also showed an improvement in health distress, which decreased by 0.743 from baseline (measured on a $0-5$ point scale) as compared to the control group, which showed a decrease in health distress of $0.069(P<0.0001) .{ }^{35}$

Participants also showed improvement in overall fatigue, measured on a $1-10$ point scale. The treatment group had a decrease of 1.24 in fatigue from baseline, as compared with the control group, which showed a mean fatigue decrease of $0.376(P=0.001)$. Pain and discomfort, measured on a $0-10$ point scale, also decreased significantly in the treatment 
group, which reported a 1.26 decrease from baseline, in comparison with the control group, which reported a decrease of 0.463 ( $P=0.014)$. Role function, measured on a $0-7$-point scale, also improved among the treatment group, which showed an improvement of 0.392 , as compared to the control group, which improved by $0.108(P=0.0003) .{ }^{35}$

The participants also showed improvement on the health behavior outcome measures. The treatment group showed a 63.7-minute increase in exercise per week from baseline, as compared with a 31-minute increase among the control group $(P=0.001)$. The treatment group also showed a 0.7 improvement in communication with a physician on a 1-3-point scale from baseline, as compared with the control group, which showed an improvement of $0.223(P<0.0001)$. Finally, the treatment group showed a 0.833 improvement in mental stress management from baseline, measured as times per week, as compared to a decrease of 0.213 among the control group $(P<0.0001){ }^{35}$

The treatment group also showed improvement in the health care utilization outcome measures. The number of physician visits within the past four months decreased by 0.475 among the treatment group from baseline, as compared to a decrease of 0.034 in the control group $(P<0.021)$. The number of visits to the emergency department in the past four-months also decreased among the treatment group by 0.083 from baseline, as compared to an increase of 0.101 in the control group $(P=0.002) .^{35}$

The study authors also compare the mean self-efficacy, health status, health behavior, and health care utilization of the participants in the intervention group at baseline and after one year. They found a mean improvement in self-efficacy of 1.17 from baseline among participants of the intervention group $(P<0.001) .{ }^{35}$ They also found significant improvement in health status outcomes. Among the participants in the program, the researchers report an improvement of 0.277 in self-reported health status $(P<0.0001)$, a 0.788 decrease in health distress $(P<0.0001)$, a 1.34 decrease in fatigue $(P<0.0001)$, a 1.31 decrease in pain or physical discomfort $(P<0.0001)$, and an improvement in role function of $0.394(P<0.0001)$ after one year. ${ }^{35}$

The study authors also report improvement in study participants' health behaviors one year after completion of the program. Mean exercise increased by 59 minutes per week $(P<0.0001)$, communication with a physician improved by $0.732(P<0.0001)$, mental stress management improved by $0.621(P<0.0001)$, and the percentage of participants using tobacco decreased by $0.011(P=0.05)$. The study authors also report that participants in the study reported 0.119 fewer ER visits within the past four months one year after completion of the study $(P=0.01) .{ }^{35}$

\section{Program 3}

The "Women Take PRIDE" program ${ }^{36}$ was developed based on an earlier chronic disease self-management intervention called "Take PRIDE". ${ }^{37}$ The "Women Take PRIDE" program aimed to enhance overall disease self-management among women aged 60 years and older diagnosed with CVD. Those assigned to the treatment group received an intervention based on social cognitive theory, ${ }^{28}$ with steps of self-regulation including problem identification, researching one's routine, identifying a management goal, developing a plan to reach it, expressing one's reactions and establishing rewards for goal achievement (PRIDE). ${ }^{37}$

The original "Take PRIDE" program ${ }^{37}$ used social cognitive theory to tailor a self-management program to the needs of older adults with heart disease. The two goals of this program were to raise the awareness of program participants to the self-regulation, and to assist participants in improving their health behaviors and their social and physical environments in order to improve their self-management. Program participants attended four weekly group meetings of two hours duration, with six to eight individuals per group. The program involved watching a video about effective self-management, receiving instruction from a health educator on health behaviors, identifying a problem area as a target for improvement, and using the tools discussed in the program to improve health behaviors. Participants were also encouraged to observe their own health behaviors and compare them with the health behaviors discussed during the program, as well as to observe the changes in their health behaviors throughout the program. ${ }^{37}$

An evaluation of this program included 246 individuals randomly assigned to the control and treatment groups. At the end of the four "Take PRIDE" meetings, participants were asked to complete a questionnaire. Members of both the control and treatment groups completed phone interviews at baseline, two months after the completion of the program, and 12 months after completion. The study authors measured self-efficacy by asking program participants to self-report levels of confidence in their health regimen on a scale of one to ten, where one is not at all confident and ten is very confident. Only those who selected their diet as their area of difficulty on which to focus for the duration of the program had a statistically significant difference, from a mean diet selfefficacy of 7.93 to a two-month mean of $8.52(P=0.03) .{ }^{37}$

In the "Women Take PRIDE" program, a total of 443 women participated in the study, 227 in the control 
group and 216 in the intervention group. Participants in the intervention met in groups of six to eight for 2.5 hours during four consecutive weeks. Based on the heart regimen prescribed by their physicians, participants selected a management area that had been problematic for them, such as exercise, medication adherence, or diet. Facilitated by a health education and peer leader, the women learned the steps of the PRIDE process while applying the process to their individual disease management problem. Instructional materials tailored to women's interests were also provided to the participants. These included a workbook, videotape, and self-monitoring tools such as a pedometer. ${ }^{36}$

Another component of the "Women Take PRIDE" study assessed the impact of the program on use of hospital services and compared hospital cost savings from reduced use of services with the cost of offering the program. ${ }^{36}$ Patients in this study were randomly assigned to the intervention group or the control group, which received the usual standard of care. The study authors tested the difference in impact between the intervention and control group, using Wilcoxon's two-sample tests for continuous variables and the $\chi^{2}$ test for dichotomous variables. The study authors then used multiple regression models to examine the effects of the intervention, controlling for baseline utilization, health status, demographic variables, and hospital site. Hospital care utilization and cost were examined using hospital admissions, hospital inpatient days and hospital inpatient costs as dependent variables, as well as the number and costs of emergency department visits. $^{36}$

The researchers found that participants of the "Women Take PRIDE" study experienced $46 \%$ fewer hospital inpatient days than women in the control group $(P=0.03)$. Additionally, women in the program incurred approximately $49 \%$ lower inpatient charges than control group women $(P=0.10)$. Savings in inpatient charges were approximately $\$ 3,200$ per participant per year. Women who participated in the program also experienced $41 \%$ fewer heart-related hospital admissions than women in the control group $(P=0.05)$. Participants also incurred an in-patient cost that was $44 \%$ less than the control group $(P=0.04)$ and $61 \%$ fewer in-patient days than those in the control group $(P=0.01)$. In comparison, the researchers noted that the direct costs of delivering the four-week program to a group of eight participants were approximately $\$ 130$ per patient. The study authors estimated that the ratio of total cost to expenditure savings was 1 to $5 .{ }^{36}$ Several subsequent studies also evaluated the impact of this program and also found positive results. ${ }^{36}$

\section{Program 4}

Another study identified in our review ${ }^{38}$ examined the effect of CR on exercise self-efficacy (defined as confidence in one's ability to exercise under certain conditions), motivational readiness for exercise, and decisional balance for exercise adoption for patients with CVD. The theoretical foundation of this program was the transtheoretical model (TTM) of motivational readiness, which conceptualizes the process of behavior change through a progression of "stages" of readiness. ${ }^{39}$

$\mathrm{CR}$ is a medically supervised phase II program that combines physical exercise with behavior modification, nutrition information and other educational and support mechanisms for cardiac patients. This program was a 12 -week exercise regimen administered by a nurse, an exercise physiologist and a cardiologist. The participants met three times per week for one hour and 15 minutes per session for the exercise portion of the program, and weekly for educational classes on medication management, cardiac risk factors, guidelines for home activity, stress management and nutrition planning. ${ }^{38}$

Forty-six patients aged 39-74 years completed the baseline, posttreatment and follow-up analyses. Exercise prescriptions were calculated from the maximum heart rate achieved during the patient's preliminary stress test. Physical activity during the intervention was assessed using a questionnaire and through consultation with the CR staff. Self-efficacy was evaluated using a five-item instrument and a five-point Likert scale on which participants indicated their confidence that they could remain physically active outside of CR under several specific conditions such as inclement weather. Stage of readiness for exercise adoption was assessed via five items concerning current exercise practices and intention to change. Internal consistency for this measure is $0.82 .{ }^{38}$

The study authors used $\chi^{2}$ tests to assess the number of individuals within each stage of readiness for exercise, and analysis of variance (ANOVA) with Bonferroni corrections to examine differences in self-efficacy, decisional balance, and the behavioral and cognitive processes of change across the states of readiness for exercise at baseline and 12 weeks later (posttreatment). The study showed that CR produced significant gains in both exercise-related selfefficacy and motivational readiness for exercise between baseline and posttreatment. Participants reported a significant increase in self-efficacy for exercise, $F(1,45)=15.4$, $(P<0.01)$ as compared to baseline, the time spent in exercise, $F(1,45)=6.1(P<0.01)$ and a reduction in ratings of the barriers to exercise, $F(1,45)=3.9(P<0.05) .{ }^{40}$ Other evaluations of the role of self-efficacy in CR have also found a positive correlation. ${ }^{41,42}$ 


\section{Program 5}

The final program ${ }^{43}$ included in the review is a disease management program developed for low literacy patients with heart failure. The developers of the program incorporated educational materials targeted at low-literacy patients into a disease management program to assist cardiac patients with adherence to disease management and to improve self-efficacy. ${ }^{43}$

The participants received a picture-based educational booklet using simple language to underscore each selfmanagement lesson with associated graphics to reinforce understanding. Using a color-coded system, the developers of the program emphasized the signs and symptoms associated with worsening heart failure and indicated when a change in diuretic dosing was needed. The materials instructed patients to adjust their diuretic dosage based on an algorithm specific to the needs of the individual patient. The materials also focused on supporting patients' adherence to their regimen. The researchers tested the materials with a two-hour focus group of four patients and then performed individual cognitive response interviews with four more patients. ${ }^{43}$

Twenty-five patients were enrolled in the preliminary study testing the program. The intervention included a one-hour educational session with a pharmacist or health educator during a regular clinic visit. Patients were given the educational booklet and a digital scale. The health educator then made seven follow-up calls with each patient designed to reinforce the educational session. ${ }^{43}$

The main outcome measures of the study were literacy, heart failure knowledge and patient self-efficacy. The literacy measure was based on an established test of functional health literacy and the heart failure knowledge measure was assessed using a newly developed 15-question tool administered verbally to each patient. The study authors developed an eight-question instrument to determine patients' selfefficacy focusing on specific activities related to self-care in the intervention. The participants responded using a fourpoint Likert-type scale ranging from "I know I cannot" to "I know I can" yielding a score from 8 to 32 . The researchers also assessed behavior change by asking the patients to selfreport the frequency of weight assessment and by filling out $\log$ sheets. $^{43}$

The researchers found that 12 weeks after the program, $100 \%$ of participants self-reported weighing themselves everyday or several times per week, as compared to $32 \%$ at baseline. The researchers also found a statistically significant mean $d$ in self-efficacy of 1.2 on a 32-point scale $(95 \%$ confidence interval [CI]: 0.06-2.3). Using the Minnesota Living with Heart Failure questionnaire to assess heart failure symptoms, the researchers also found that at three months, the statistically significant mean decrease in heart failure symptoms was 9.9 on a scale or $0-105$, with higher scores representing worse symptoms (95\% CI:-0.5--19.2), which they stated represented a clinically important improvement. In previous studies, a 10-point improvement in heart failure quality-of-life based on their changing scores. ${ }^{43}$

\section{Discussion}

The interventions described above represent a spectrum of disease management programs evaluated over the past fifteen years. While the facets of each program are distinct and the processes of development, implementation, and assessment vary widely between each program, several commonalities among the programs are worth noting.

Most significantly for this review, each of these programs recognizes the value of self-efficacy in patients' adherence to treatments and ability to manage their disease. The programs quantitatively measured self-efficacy of patients after the intervention and compared their scores with baseline values and/or with those of the control group. Each of the study authors note that patients' confidence in their ability to manage their disease and perform daily activities was an important aspect of the success of disease management programs, and each of the study results discussed above support this claim.

Another commonality among the studies was a multidisciplinary approach. Each study had several components offering different kinds of support including written materials, follow-up phone calls from group leaders or clinicians, peer group support and/or exercise materials. Several programs also provided self-assessment and self-management aids, such as emphasizing the importance of tracking weight gain and loss. From the results, this multidisciplinary approach seemed to improve the patients' self-efficacy by reaching each patient at his or her current status and allowing the patients to apply the materials to their individual needs.

This was particularly clear in the "Women Take PRIDE" study, in which patients were asked at the outset to designate a problem to which they would apply the program's tools. ${ }^{44}$ This individualized approach tailored the multidisciplinary activities to the specific needs and goals of each patient while simultaneously fostering the peer support of a group program with low direct costs. ${ }^{36}$

Another common theme among these programs is a foundation of well-developed theories of human behavior and decision making. While there was some variation in the theoretical foundation of the study designs, each program 
was developed using theories of human behavior and disease management. These theories included Bandura's theory of self-efficacy ${ }^{28}$ and the TTM of behavior change. ${ }^{39}$ The underlying foundation of behavioral theory seemed to be an important driving force for these programs in reaching their patients at their current level of self-efficacy and assisting them with their disease management from that point, as well as seeking to improve patient confidence in their abilities to self-manage. These results suggest that CVD management programs driven by behavioral theory that emphasize patient self-efficacy can have important clinical impacts and may also be a mechanism for reducing unnecessary health services utilization and health care costs.

Several significant limitations should be noted. In each program evaluated, the intervention was performed on a voluntary basis. This may cause some self-selection bias towards patients that already have higher self-efficacy. Additionally, in some cases compensation was given to the patients for participating in the study. This could also bias the results towards those for whom compensation is most significant. The studies considered in this review are also limited to studies performed in the United States. This may mean that important international studies were overlooked during the search process. And finally, while the studies described here show the positive correlation between self-efficacy and increased clinical outcomes, the direction of causality is unclear. While it is unlikely that self-efficacy could worsen clinical outcomes, it is also likely that better clinical outcomes could increase the self-efficacy of the patient in addition to improved self-efficacy increasing clinical outcomes.

\section{Implications for practice}

The literature shows the importance of patient engagement and self-efficacy as mechanisms for self-management of CVD. Future development of self-management programs for CVD should emphasize and seek to enhance patient selfefficacy using theories of behavioral change. Additionally, these interventions should be tailored to the needs of many different populations. Given their higher tendency to face challenges managing their disease, programs should target low-income patients, minority groups and those with limited English proficiency. Finally, more research should be conducted on the role of self-efficacy in self-management programs and the most effective methods for disease management interventions to improve patient self-efficacy.

\section{Disclosure}

The authors report no conflicts of interest in this work.

\section{References}

1. Centers for Disease Control. Health, United States, 2009. Available from: http://www.cdc.gov/nchs/hus.htm. Accessed May 10, 2010.

2. Lloyd-Jones D, Adams R, Carnethon M, et al. Heart Disease and Stroke Statistics 2009 Update: A Report from the American Heart Association Statistics Committee and Stroke Statistics Subcommittee. Circulation. 2009;119:e1-e161.

3. World Health Organization. Cardiovascular Disease Atlas: World Data Table. World Health Organization Global Infobase. Database online. Accessed Jul 16, 2010. Available from: https://apps.who.int/infobase/report.aspx

4. Centers for Disease Control. Prevalence of heart disease - United States, 2005. MMWR Morb and Mortal Wkly Rep. 2007;56(6):113-118.

5. Ara S. A literature review of cardiovascular disease management programs in managed care populations. J Manag Care Pharm. 2004;10(4):326-344.

6. Sarkar U, Ali S, Whooley MA. Self-efficacy and health status in patients with coronary heart disease: findings from the heart and soul study. Psychosom Med. 2007;69:306-312.

7. Sol BGM, van der GraafY, van der Bifl JJ, Goessens BGN, Visseren FLJ. Self-efficacy in patients with clinical manifestations of vascular diseases. Patient Educ Couns. 2006;61:443-448.

8. RozanskiA. Integrating psychologic approaches into the behavioral management of cardiac patients. Psychosom Society. 2005;67:S67-S73.

9. Lorig KR, Holman HR. Self-management education: history, definition, outcomes, and mechanisms. Ann Behav Med. 2003;26(1):1-7.

10. Corbin J, Strauss A. Unending work and care: managing chronic illness at home. San Francisco: Jossey-Bass, 1988.

11. Lorig KR, Sobel DS, Stewart AL, et al. Evidence suggesting that a chronic disease self-management program can improve health status while reducing hospitalization: a randomized trial. Med Care. 1999;37:5-14.

12. Lorig KR, Ritter P, Stewart AL, et al. Chronic disease self management program: 2-year health status and health care utilization outcomes. Med Care. 2001;39(11):1217-1223.

13. Lorig KR, Ritter PL, Saurent DD, Plant K. Internet-based chronic disease management: a randomized trial. Med Care. 2006;44(11):964-971.

14. Gordon K, Smith F, Dhillon S. Effective chronic disease management: patient's perspectives on medication-related problems. Patient Educ Couns. 2007;65:407-415.

15. Haynes RB, Yao X, Degani A, Kripalani S, Garg A, McDonald HP. Interventions to enhance medication adherence. Chochrane Database of Syst Rev. 2005;(4):CD000011.

16. Kripalani S, Henderson LE, Chiu EY, Robertson R, Kolm P, Jacobson TA. Predictors of medication self-management skill in a low-literacy population. J Gen Intern Med. 2006;21:852-856.

17. Jerant AF, con Frederichs-Fitzwater MM, Moore M. Patients' perceived barriers to active self-management of chronic conditions. Patient Educ Couns. 2005;57:300-307.

18. Blustein J, Valentine M, Mean H, Regenstein M. Race/Ethnicity and patient confidence to self-manage cardiovascular disease. Medical Care. 2008;46(9):924-929.

19. Clark NM, Dodge JA. Exploring self-efficacy as a predictor of disease management. Health Educ and Behav. 1999;26(72):72-89.

20. Sullivan MD, LaCroix AZ, Russo J, Katon WJ. Self-efficacy and self-reported functional status in coronary heart disease: a six-month prospective study. Psychosom Med. 1998;60:473-478.

21. Newman S, Engaging patients in managing their cardiovascular health. Heart. 2004;90:iv9-iv13.

22. Weiss BD, Hart G, McGee DL, D'Estelle S. Health status of illiterate adults: relation between literacy and health status among persons with low literacy skills. J Am Board Fam Pract. 1992;5: 257-264.

23. Baker DW, Wolf MS, Feinglass J, Thompson JA, Gazmararian JA, Huang J. Health literacy and mortality among elderly persons. Arch Intern Med. 2007;167(14):1503-1509.

24. Mensah GA, Mokdad AH, Ford ES, Greenlund KJ, Croft JB. State of disparities in cardiovascular health in the United States. Circulation. 2005;111:1233-1241. 
25. Kogan MD, Kotelcuck M, Alexander GR, Johnson WE. Racial disparities in reported prenatal care advice from health care providers. Am J of Public Health. 1994;84(1):82-88.

26. Greene J, Yedida MJ, Take Care to Learn Evaluation Collaborative. Provider behaviors contributing to patient self-management of chronic illness among underserved populations. J Health Care Poor and Underserved. 2005; 16:808-824.

27. Cooper-Patrick L, Gallo JJ, Gonzales JJ, et al. Race, gender, and partnership in the patient-physician relationship. JAMA. 1999;282(6):583-589.

28. Bandura A. Self-efficacy mechanism in human agency. American Psychologist. 1982;37(2):122-147.

29. Dongbo F, Dine Y, McGowan P, Fu H. Qualitative evaluation of Chronic Disease Self Management Program (CDSMP) in Shanghai. Patient Educ Couns. 2006;61(3):389-396.

30. Elzen H, Slaets JPJ, Snijders TAB, Steverink N. Evaluation of the chronic disease self-management program (CDSMP) among chronically ill older people in the Netherlands. Soc Sci Med. 2007;64(9): 1832-1841.

31. Goeppinger J, Armstrong B, Schwartz T, Ensley D, Brady TJ. Self-management education for persons with arthritis: Managing comorbidity and eliminating health disparities. Arthritis Rheum. 2007;57(6):1081-1088.

32. Chan SCC, Siu AMH, Poon PKK, Chan CCH. Chronic disease selfmanagement program for Chinese patients: a preliminary multi-baseline study. Int J of Rehab Res. 2005:28(4):351-354.

33. Griffiths C, Motlib J, Azad A, et al. Randomised controlled trial of layled self-management programme for Bangladeshi patients with chronic disease. Br J Gen Pract. 2005;55(520):831-837.

34. Lorig K, Stewart A, Ritter P, González V, Laurent D, Lynch J. Outcome measures for health education and other health care interventions. Thousand Oaks, CA: Sage Publications; 1996.
35. Lorig KR, Ritter PL, Gonzalez VM. Hispanic chronic disease selfmanagement: A randomized community-based outcome trial. Nursing Research. 2003;52(6):361-369.

36. Wheeler JRC, Janz NK, Dodge JA. Can a disease self-management program reduce health care costs? The case of older woman with heart disease. Med Care. 2003;41(6):706-715.

37. Clark NM, Janz NK, Dodge JA, Sharpe PA. Self-regulation of health behavior: the "take PRIDE" program. Health Educ $Q$. 1992;19(3):341-354.

38. Bock BB, Albrecht AE, Traficante RM, et al. Predictors of exercise adherence following participation in a cardiac rehabilitation program. Int J Beh Med. 1997;4(1):60-75.

39. Prochanska JO, DiClemente CC. Stages and processes of self-change in smoking: Toward an integrative model of change. J Consult Clin Physchol. 1983;51:390-395.

40. Zarnke KB, Feagan BG, Mahon JL, Feldman RD. A randomized study comparing a patient-directed hypertension management strategy with usual office-based care. The Am J Hypertens. 1997;10:58-67.

41. Carloson JJ, Norman GJ, Feltz DL, Franklin BA, Johnson JA, Locke SK. Self-efficacy, psychosocial factors, and exercise behavior in traditional versus modified cardiac rehabilitation. J Cardiopulmonary Rehabil. 2001;21(6):363-373.

42. Izawa KP, Watanabe S, Omiya K, et al. Effect of the self-monitoring approach on exercise maintenance Turing cardiac rehabilitation: A randomized, controlled trial. American Journal of Physical Medicine and Rehabilitation. 2005;84:313-321.

43. deWalt DA, Pgnone M, Malone R, et al. Development and pilot testing of a disease management program for low literacy patients with heart failure. Patient Educ Couns. 2004;55:78-86.

44. Clark NM, Janz NK, Becker MH, et al. Impact of self-management education on the functional health status of older adults with heart disease. Gerontologist. 1992;32(4):438-443.
Patient Intelligence

\section{Publish your work in this journal}

Patient Intelligence is an international, peer-reviewed, open access journal that characterizes and measures the central role of patient behavior and intention in optimizing healthcare management in all areas of disease and complaint types. An improved understanding of patient intelligence coupled with predictive analysis helps an organization contribute more effectively to achieving better outcomes.

Submit your manuscript here: http://www.dovepress.com/patient-intelligence-journal

\section{Dovepress}

The journal is characterized by the rapid reporting of reviews, original research, methodologies, analytics, modeling, clinical studies and patient surveys across all disease areas. The manuscript management system is completely online and includes a very quick and fair peer-review system. Visit http://www.dovepress.com/ testimonials.php to read real quotes from published authors. 